\title{
Selection of the Appropriate Control Group Is Essential in Evaluating the Cytokine Storm in COVID-19
}

\author{
ALICE G. VASSILIOU ${ }^{1}$, IOANNA DIMOPOULOU ${ }^{1}$, EDISON JAHAJ ${ }^{1}$, CHRYSI KESKINIDOU ${ }^{1}$, \\ ZAFEIRIA MASTORA ${ }^{1}$, STYLIANOS E. ORFANOS ${ }^{1,2}$ and ANASTASIA KOTANIDOU ${ }^{1}$ \\ ${ }^{1}$ First Department of Critical Care Medicine \& Pulmonary Services, School of Medicine, \\ National and Kapodistrian University of Athens, Evangelismos Hospital, Athens, Greece; \\ ${ }^{2}$ Second Department of Critical Care, School of Medicine, \\ National and Kapodistrian University of Athens, Attikon Hospital, Athens, Greece
}

\begin{abstract}
Background/Aim: Lately, studies have reported contradicting results on the cytokine storm seen in criticallyill COVID-19 patients. Depending on the control group used, cytokines have been found to be higher, similar or even lower in COVID-19 compared to critical illnesses associated with elevated cytokine concentrations. However, most of these studies do not take into account critical illness severity. Hence, we decided to compare cytokine levels in critically-ill COVID-19 patients and critically-ill patients of a general intensive care unit (ICU), who did not have sepsis or septic shock, but had an equal disease severity. Patients and Methods: Interleukin (IL)-6, IL-8, IL-10 and tumour necrosis factor- $\alpha(T N F-\alpha)$ were measured on ICU admission in mechanically ventilated, COVID-19 $(N=36)$ and non-COVID$19(N=30)$ patients, who had not received dexamethasone, and had equal critical illness severity. Non-COVID-19 patients did not have sepsis or septic shock. Results: In our case control study, circulating IL-6 and IL-10 were lower, while TNF- $\alpha$ and IL-8 levels were higher in critically-ill COVID-19 patients, compared to critically-ill non-COVID19 patients. Conclusion: It is difficult to infer whether the cytokine storm seen in COVID-19 differs from other critical conditions. It is important to recognize that the conclusions of related studies may depend on control group selection.
\end{abstract}

This article is freely accessible online.

Correspondence to: Prof. Anastasia Kotanidou, MD, $1^{\text {st }}$ Department of Critical Care Medicine and Pulmonary Services School of Medicine, National and Kapodistrian University of Athens, Evangelismos Hospital, 45-47 Ipsilantou St, 106 76, Athens, Greece. Tel: +30 2107243320, Fax: +30 2107216503, e-mail: akotanid@med.uoa.gr

Key Words: COVID-19, cytokine storm, critically-ill, ICU.
Excellent reports and meta-analyses are being published lately questioning the cytokine response seen in critically-ill COVID-19 patients. Depending on the comparison group used, results are contradicting. Interleukin (IL)-6 has been shown to be lower in critical COVID-19 compared to septic shock with or without ARDS $(1,2)$, and hypo- and hyperinflammatory ARDS $(3,4)$, or similar to trauma patients (1); IL-8 has been shown to be lower in critical COVID-19 compared to septic shock with or without ARDS (1), and hyper-inflammatory ARDS, but similar to hypoinflammatory ARDS (3). Tumour necrosis factor- $\alpha$ (TNF- $\alpha$ ) has been shown to be lower or similar to sepsis with or without ARDS, but higher compared to trauma $(1,3)$. Other cytokines, such as IL-10, have not been sufficiently investigated, while one report showed no differences between COVID-19 and sepsis in 76 cytokines (5). However, the above reports do not account for differences in critical illness severity, as expressed by the acute physiologic assessment and chronic health evaluation II (APACHE II). and sequential organ dysfunction assessment (SOFA) scores, do not take into account dexamethasone administration, or have compared cytokine levels measured by different platforms. Since it seems that the control group matters, we decided to compare cytokine levels in critically-ill COVID19 patients and critically-ill patients of a general intensive care unit (ICU), who did not have sepsis or septic shock, but had an equal disease severity. None of the patients studied had received dexamethasone.

\section{Patients and Methods}

This prospective observational case control study was carried out in one academic multi-disciplinary ICU. All procedures performed were in accordance with the ethical standards of the institutional and/or national research committee, and with the 1964 Helsinki Declaration and its later amendments. The study was approved by the Hospital Research Ethics Committee (129/19-3-2020). Informed consent was obtained by patients' next-of-kin. ICU admission 
Table I. Patient characteristics on ICU admission.

\begin{tabular}{|c|c|c|c|}
\hline Parameters & $\begin{array}{l}\text { Critically-ill COVID-19 } \\
\qquad(\mathrm{N}=36)\end{array}$ & $\begin{array}{l}\text { Critically-ill non-COVID-19 } \\
\qquad(\mathrm{N}=30)\end{array}$ & $p$-Value \\
\hline Age, mean $\pm \mathrm{SD}$, years & $64 \pm 11$ & $45 \pm 19$ & $<0.0001$ \\
\hline $\operatorname{Sex}, \mathrm{N}(\%)$ & & & 0.2 \\
\hline Male & 29 (80.6) & $20(66.7)$ & \\
\hline Female & $7(19.4)$ & $10(33.3)$ & \\
\hline Comorbidities, N (\%) & $24(66.7)$ & $9(30.0)$ & 0.003 \\
\hline Diabetes & $5(13.9)$ & $3(10.0)$ & \\
\hline Hypertension & $16(44.4)$ & $4(13.3)$ & \\
\hline CAD & $4(11.1)$ & $1(3.3)$ & \\
\hline COPD & $1(2.8)$ & $1(3.3)$ & \\
\hline ARDS, $\mathrm{N}(\%)$ & $33(91.7)$ & $14(46.7)$ & $<0.0001$ \\
\hline $\mathrm{PaO} 2 / \mathrm{FiO} 2$, mean $\pm \mathrm{SD}, \mathrm{mm} \mathrm{Hg}$ & $198 \pm 85$ & $305 \pm 119$ & $<0.001$ \\
\hline APACHE II score, mean \pm SD & $15 \pm 6$ & $15 \pm 7$ & 0.9 \\
\hline SOFA score, mean \pm SD & $7 \pm 3$ & $6 \pm 3$ & 0.4 \\
\hline CRP, median (IQR), mg/dl & $11.8(5.3-19.8)$ & $4.9(2.4-10.0)$ & 0.004 \\
\hline White blood cell count, mean $\pm \mathrm{SD}$, cells $/ \mu 1$ & $10200 \pm 4700$ & $12400 \pm 5200$ & 0.08 \\
\hline IL-6, median (IQR), pg/ml & $35.0(0.7-199.4)$ & $257.9(126.4-722.1)$ & $<0.0001$ \\
\hline IL-8, median (IQR), pg/ml & $175.2(57.6-412.3)$ & $75.6(37.2-167.8)$ & 0.03 \\
\hline IL-10, median (IQR), pg/ml & $3.9(3.9-35.7)$ & $175.3(82.5-292.2)$ & $<0.0001$ \\
\hline TNF- $\alpha$, median (IQR), pg/ml & $81.3(32.0-144.5)$ & $46.5(30.1-55.1)$ & 0.009 \\
\hline Mortality, N (\%) & $10(27.8)$ & $5(16.7 \%)$ & 0.4 \\
\hline
\end{tabular}

Data are expressed either as number of patients $(\mathrm{N})$ and percentages of totals $(\%)$, mean $\pm \mathrm{SD}$, or median (IQR), as appropriate. Two-group comparisons were performed using the Student's t-test or the non-parametric Mann-Whitney test. Associations between qualitative variables were examined by the Chi-square test. All parameters were estimated within the first 24 hours post ICU admission. APACHE: Acute physiology and chronic health evaluation; ARDS: acute respiratory distress syndrome; CAD: coronary artery disease; COPD: chronic obstructive pulmonary disease; COVID-19: coronavirus disease 19; CRP: C-reactive protein; ICU: intensive care unit; IL: interleukin; IQR: interquartile range; SD: standard deviation; SOFA: sequential organ failure assessment; TNF- $\alpha$ : tumour necrosis factor- $\alpha$.

(within 24 hours) serum concentrations of IL-6, IL-8, IL-10, and TNF- $\alpha$ were determined by ELISA (R\&D Systems Inc., Minneapolis, MN, USA), by the same researcher, in 36 consecutive critically-ill COVID-19 patients and 30 consecutive critically-ill non-COVID-19 patients. SARS-CoV-2 infection was diagnosed by real-time reverse transcription PCR (RT-PCR) in nasopharyngeal swabs. The COVID-19 patients suffered from pneumonia and were directly admitted to the ICU of the Hospital, from March 22 to August 3 2020. Only patients receiving dexamethasone prior to blood sampling were excluded from the study. There were no other exclusion criteria. The critically-ill non-COVID-19 patients were a subset of a larger, previously studied, in a different non cytokinerelated context, cohort of critically-ill patients (6). Patients with sepsis on or within the first 24 hours of ICU admission, no need for intubation, brain death, end-stage cancer, total ICU stay $<3$ days, re-admission or transfer from another ICU, contagious diseases, oral or inhaled intake of corticosteroids for a period of more than one month, and use of immunosuppressive medication were excluded.

Data were expressed as mean \pm standard deviation (SD) for normally distributed variables, median with interquartile range (IQR) for variables with skewed distribution and as count (\%) when categorical. Two-group comparisons were performed using the Student's $t$-test or the non-parametric Mann-Whitney test. Associations between qualitative variables were examined by the chi-square test. All aforementioned analyses were performed using the GraphPad software (San Diego, CA, USA). All $p$-values are two-sided; $p<0.05$ was considered statistically significant.

\section{Results and Discussion}

The critically-ill non-COVID-19 patients consisted of trauma ( $\mathrm{N}=16)$, surgical $(\mathrm{N}=8)$ and medical (CNS-related pathologies, $\mathrm{N}=6$ ) cases. As seen in Table I, there were no differences in disease severity between the two groups. COVID-19 patients exhibited the first symptoms $6 \pm 2$ days prior to admission. In our cohort, circulating IL-6 and IL-10 were lower in critically-ill COVID-19 patients, while TNF$\alpha$ and IL- 8 levels were higher, compared to critically-ill nonCOVID-19 patients (Table I and Figure 1).

In this report, not all cytokines measured in critically-ill COVID-19 patients were lower compared to critically-ill nonCOVID-19 subjects with the same disease severity. These preliminary results agree only in part with those presented in the aforementioned recently published reports (1, 3-5). Overall, it seems that there is a complexity of cytokine responses in critically-ill patients. This could explain why treatments with anti-cytokine agents have failed in general ICU patients, despite the fact that in mixed ICU patients cytokine concentrations on ICU admission act as independent outcome predictors (7). While it is clear that in COVID-19 circulating cytokines are high, it remains currently unanswered whether their levels exceed those of other 


\section{A}
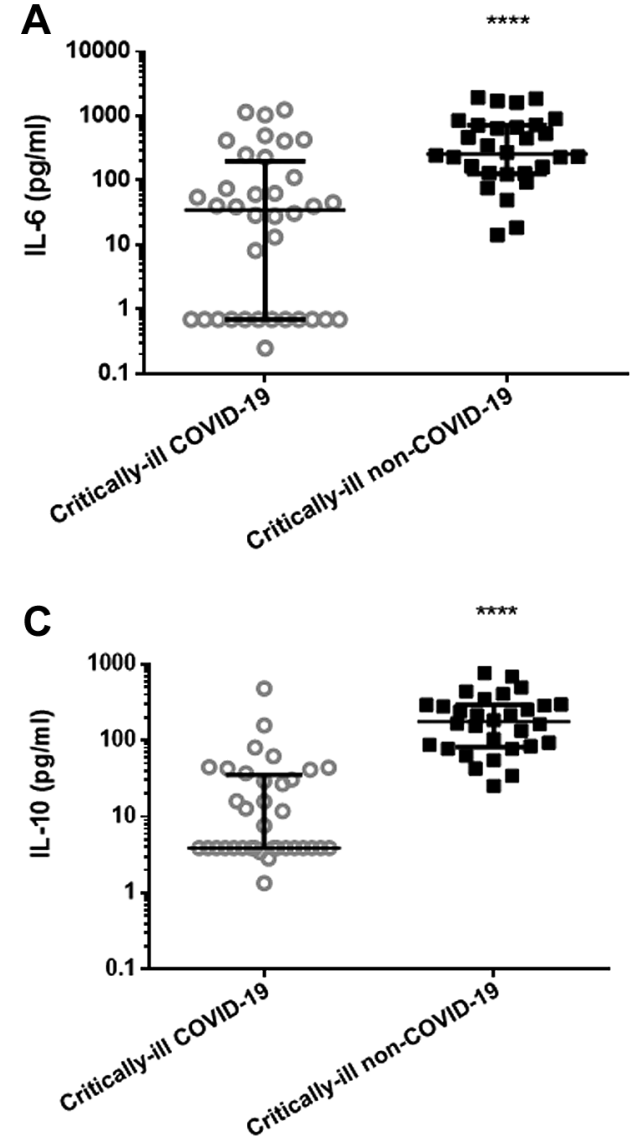
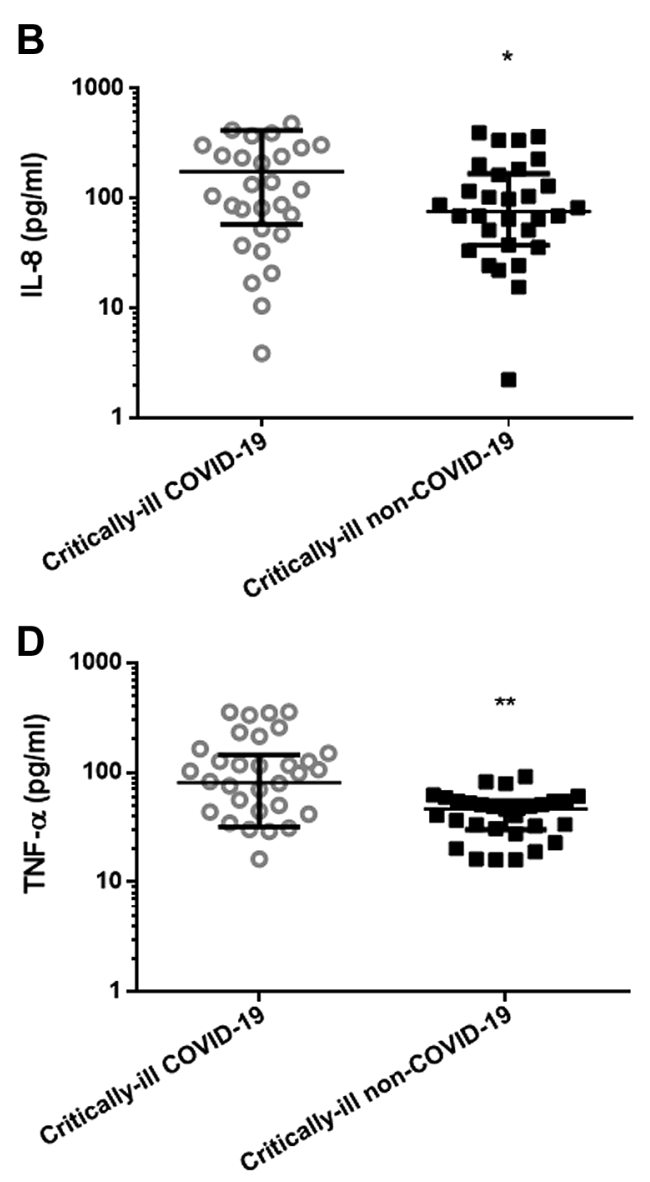

Figure 1. Cytokine levels in critically-ill COVID-19 and non-COVID-19 patients. IL-6 (A), IL-8 (B), IL-10 (C), and TNF- $\alpha$ (D) were measured on ICU admission in 36 critically-ill COVID-19 patients and 30 critically-ill non-COVID-19 patients. Two-group comparisons were performed using the non-parametric Mann-Whitney test for skewed data. Data are presented as scatter plots showing median and interquartile range. Line in the middle, median value; upper and lower lines, $25^{\text {th }}$ to $75^{\text {th }}$ centiles. ${ }^{*} p<0.05, * * p<0.01, * * * *<<0.0001$. COVID-19: Coronavirus disease 19, IL: Interleukin, TNF- $\alpha$ : tumour necrosis factor- $\alpha$.

critically-ill patients. The results of studies have suggested that the term "cytokine storm" does not appropriately describe COVID-19-induced organ dysfunction (1-5). It is important to recognize that the conclusions of related studies may depend on control group selection. Critically-ill COVID19 patients exhibit a hyper-inflammatory response and develop organ failures, but the underlying mechanisms have not been elucidated. As in other syndromes of critical illness, the role of elevated inflammatory cytokines in the pathobiology of COVID-19 remains unclear. It is of outmost importance to correctly recognise the subset of COVID-19 patients who might benefit from cytokine-targeting therapy, such as IL-6 inhibitors, or drugs that inhibit cytokine expression (8). Limitations of the current investigation include the single-centre nature of the study, the small sample size, and the fact that four cytokines were measured, capturing only a part of the whole picture.

\section{Conflicts of Interest}

The Authors declare that there is no conflict of interest in regard to this study.

\section{Authors' Contributions}

$\mathrm{AGV}, \mathrm{EJ}, \mathrm{CK}$ and $\mathrm{ZM}$ collected specimens and data, designed and conducted experiments and drafted the manuscript; ID, SEO and AK supervised patient enrolment, oversaw the study, contributed to its design, and edited the manuscript. All Authors read and approved the final manuscript.

\section{Acknowledgements}

This work was funded by the non-profit institute THORAX Research Centre for Intensive and Emergency Thoracic Medicine, Athens, Greece. 


\section{References}

1 Kox M, Waalders NJB, Kooistra EJ, Gerretsen J and Pickkers P: Cytokine levels in critically ill patients with covid-19 and other conditions. JAMA 324(15): 1565-1567, 2020. PMID: 3288065. DOI: $10.1001 /$ jama.2020.17052

2 Hutchings SD, Watchorn J, Trovato F, Napoli S, Mujib SF, Hopkins $\mathrm{P}$ and McPhail M: Microcirculatory, endothelial and inflammatory responses in critically ill patients with covid-19 are distinct from those seen in septic shock: A case control study. Shock, 2020. PMID: 33021572. DOI: 10.1097/SHK.0000000000001672

3 Leisman DE, Ronner L, Pinotti R, Taylor MD, Sinha P, Calfee CS, Hirayama AV, Mastroiani F, Turtle CJ, Harhay MO, Legrand M and Deutschman CS: Cytokine elevation in severe and critical covid-19: A rapid systematic review, meta-analysis, and comparison with other inflammatory syndromes. Lancet Respir Med 8(12): 1233-1244, 2020. PMID: 33075298. DOI: 10.1016/S2213-2600(20)30404-5

4 Sinha P, Matthay MA and Calfee CS: Is a "cytokine storm" relevant to covid-19? JAMA Intern Med 180(9): 1152-1154, 2020. PMID: 32602883. DOI: 10.1001/jamainternmed.2020.3313

5 Wilson JG, Simpson LJ, Ferreira AM, Rustagi A, Roque J, Asuni A, Ranganath T, Grant PM, Subramanian A, Rosenberg-Hasson Y, Maecker HT, Holmes SP, Levitt JE, Blish CA and Rogers AJ: Cytokine profile in plasma of severe covid-19 does not differ from ards and sepsis. JCI Insight 5(17): e140289, 2020. PMID: 32706339. DOI: $10.1172 /$ jci.insight. 140289
6 Vassiliou AG, Mastora Z, Orfanos SE, Jahaj E, Maniatis NA, Koutsoukou A, Armaganidis A and Kotanidou A: Elevated biomarkers of endothelial dysfunction/activation at icu admission are associated with sepsis development. Cytokine 69(2): 240-247, 2014. PMID: 25016133. DOI: 10.1016/j.cyto.2014.06.010

7 Dimopoulou I, Orfanos S, Kotanidou A, Livaditi O, GiamarellosBourboulis E, Athanasiou C, Korovesi I, Sotiropoulou C, Kopterides P, Ilias I, Kanellakopoulou K and Armaganidis A: Plasma pro- and anti-inflammatory cytokine levels and outcome prediction in unselected critically ill patients. Cytokine 41(3): 263-267, 2008. PMID: 18191577. DOI: 10.1016/j.cyto.2007.11.019

8 Pollard BS, Blanco JC and Pollard JR: Classical drug digitoxin inhibits influenza cytokine storm, with implications for covid19 therapy. In Vivo 34(6): 3723-3730, 2020. PMID: 33144490. DOI: 10.21873 /invivo.12221
Received January 8, 2021

Revised January 20, 2021

Accepted January 22, 2021 